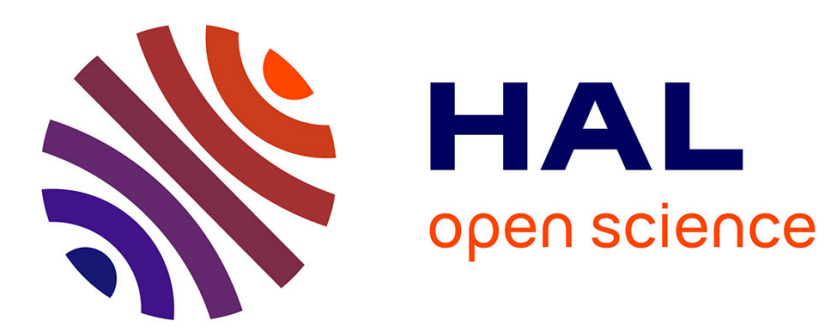

\title{
Engineered transparent emulsion to optically study particulate flows in yield stress fluids
}

\author{
Ahmadreza Rashedi, G. Ovarlez, Sarah Hormozi
}

\section{To cite this version:}

Ahmadreza Rashedi, G. Ovarlez, Sarah Hormozi. Engineered transparent emulsion to optically study particulate flows in yield stress fluids. Experiments in Fluids, 2020, 61 (2), 10.1007/S00348-019-28583. hal-02995501

\section{HAL Id: hal-02995501 \\ https://hal.science/hal-02995501}

Submitted on 10 Nov 2020

HAL is a multi-disciplinary open access archive for the deposit and dissemination of scientific research documents, whether they are published or not. The documents may come from teaching and research institutions in France or abroad, or from public or private research centers.
L'archive ouverte pluridisciplinaire HAL, est destinée au dépôt et à la diffusion de documents scientifiques de niveau recherche, publiés ou non, émanant des établissements d'enseignement et de recherche français ou étrangers, des laboratoires publics ou privés. 


\title{
Engineered Transparent Emulsion to Optically Study Particulate Flows in Yield Stress Fluids
}

\author{
Ahmadreza Rashedi · Guillaume Ovarlez • Sarah Hormozi
}

Received: date / Accepted: date

\begin{abstract}
We have engineered a model suspension consisting of rigid particles and yield stress fluids. The suspending fluid is an emulsion with adjustable density, rheological behavior, and refractive index. We explain the design procedure in detail. The optically transparent emulsion opens the possibility of exploring Particle Tracking/Image Velocimetry (PIV/PTV) techniques in studying dynamic flows involving particles in complex fluids. As a proof of concept, we have performed PTV to provide accurate measurements of solid volume fractions for the dispersion of particles in a Taylor-Couette cell.
\end{abstract}

Keywords Yield stress fluid suspension · Transparent emulsions $\cdot$ Particle Tacking Velocimetry

\section{Introduction}

The rheology of non-Brownian suspensions of solid particles in both Newtonian and non-Newtonian fluids has drawn much attention since there are so many industrial and daily life applications such as the oil industry, food processing, cosmetics, muds, painting, coating,

Ahmadreza Rashedi

Ohio University, 28 West Green Dr., Stocker Center 256, Athens, OH, 45701, USA

\&

Laboratiore du futur, 178 avenue du Dr. schweitzer, Pessac, 33600 , France

Tel.: +1 (740) 591-6704

E-mail: ar122512@ohio.edu

Guillaume Ovarlez

Laboratiore du futur, 178 avenue du Dr. schweitzer, Pessac, 33600 , France

Sarah Hormozi

Ohio University, 28 West Green Dr., Stocker Center 256, Athens, OH, 45701, USA etc. These suspensions show nonlinear behavior such as shear thinning, shear thickening, thixotropy, plasticity, etc. One category of these materials is yield stress fluid suspensions. Yield stress fluids are known as nonNewtonian fluids with shear thinning behavior. In other words, they do not have linear behavior when exposed to a shear rate gradient. In addition, if the applied shear stress is not sufficient, they act like solids; However, when the shear stress is larger than the yield stress of the material, it flows [1].

Characterizing the flow of particles suspended in yield stress fluids is complicated since it requires local rheological measurements due to the heterogeneity of the flow field (e.g. shear flow and solid volume fraction). As an example, shear-induced migration of particles occurs in many industrial and natural settings dealing with the importation of particles within a yield stress fluid. The engineering challenge to study the dispersion of a solid within a yield stress fluid is that the kinetics of migration in yield stress fluids are shear rate dependent. This means that we should have access to the instantaneous distribution of the solid volume fraction in order to characterize the shear-induced migration of particles in yield stress fluids.

There are experimental techniques to study the local rheology of suspensions such as Nuclear Magnetic Resonance/Imaging (NMR/I), X-ray, ultrasonic, Laser Doppler Velocimetry (LDV), and Particle Imaging/Tracking Velocimetry (PIV/PTV).

Ultrasonic velocimetry is a powerful measurement technique to study multiphase flows, which is more efficient for gas and liquid [2]. There are some limitations to this technique. It is more applicable when studying the dilute regime and does not properly work for the dense regime. In addition, fluid with a high viscosity attenuates ultrasonic waves [3-5]. 
NMR and MRI techniques have been used to investigate the flow in non-transparent suspensions from dilute to dense regimes $[6,7]$. In this technique, an applied magnetic field affects the hydrogen nuclei inside the material by targeting quantum mechanical properties[8]. The spatial resolution can be set from a micrometer to a millimeter $[9,10]$. NMR has a low temporal resolution. In other words, the material should be in a static position or in a creeping flow for an imaging sequence to have a good image resolution. This technique has been used to study particle migration and sedimentation in suspensions [11-14].

$\mathrm{X}$-ray radiography has been widely used to build detailed images of internal structures of materials. X-ray radiography can be performed from different angles and used to build up a tomographic image [15]. This method is called computed tomographic scanning (CT-scan). A CT-scan has never been used before to investigate particle dynamics in a flow since the time scale of scanning is much larger than the time scale of the flow [16]. Recently, Gholami et al. used x-ray radiography images to measure the distribution of a solid volume fraction of particles suspended in yield stress fluids flowing in a wide gap Taylor-Couette cell [17]. Since there is a symmetry in this viscometric flow, they utilized the Abel transform to compute the distribution of a solid volume fraction. The high temporal resolution of X-ray radiography makes it possible to study particle dynamics in the Taylor-Couette flow. However, the only disadvantage is that an X-ray facility is expensive, and it is not available in every fluid mechanics laboratory; therefore, there is a need to create more affordable and accessible experimental techniques.

LDV and PIV/PTV are categorized as optical techniques which require a visible laser sheet. The material needs to be optically clear to be able to use these techniques. These methods have high temporal and spatial resolution [18] and have been broadly used to study particle migration in Newtonian suspensions [19-22]. The refractive index (RI) of the solid phase and liquid phase should be accurately matched to avoid light diffraction. Therefore, the suspension needs to be transparent. The RI for Poly-methyl-methacrylate (PMMA) particles is 1.49 at a wavelength of $\lambda=532 \mathrm{~nm}$. One method is to use hydrogel materials that are RI matched with yield stress gels [23]. However, Hydrogels have some limitations. Injection molding for hydrogels cannot make them smaller than $1 \mathrm{~mm}$. In addition, hydrogels are essentially not rigid particles [23]. In this work, we introduce a transparent yield stress emulsion with the same refractive index and density as Poly-methyl-methacrylate (PMMA) particles. We have used this material to perform a typical test showing the migration of solids in a heterogeneous shear field. We utilized optical techniques which are categorized as inexpensive methods. Currently, the experimental techniques that make such a study feasible are high-resolution MRI and CT scans that are not available to all researchers. The cost of a CT scan facility is in the order of $\$ 1,000,000$; however, the cost of a PIV/PTV setup is in the order of $\$ 10,000$. Other examples for the potential use of wellcontrolled transparent yield stress fluids are sedimentation, flow characteristics in extrusion, free surface flow around large objects, and displacement of large objects. Although, studying the internal structure of suspensions in uniaxial elongation will be different which is explained in detail in the following references [24-27].

Note that the droplet size of the designed emulsion should be set to be much smaller than size of the solid particles to be able to study the effect of dispersion or migration of solids suspended in yield stress fluids.

The outline of the paper is as follows. In section 2 we give a brief discussion on emulsions and their rheology. Then, we present the process of designing a transparent emulsion. We propose methods to adjust the density and rheology of a transparent emulsion at a fixed refractive index. Finally we introduce a transparent yield stress emulsion that is RI and density matched with Poly-methyl-methacrylate (PMMA) materials. In section 3 we implement the transparent yield stress fluid suspension to study the shear-induced migration of particles in a wide gap Taylor-Couette flow.

\section{Optically engineered transparent emulsion}

\subsection{Emulsions}

Emulsions are dispersions of immiscible fluids such as oil and water. Direct emulsions are dispersions of the oil in water and inverse emulsions are the dispersions of water in oil. Dispersion of one phase into another phase increases the interfacial surface between the phases, and it needs input energy against interfacial tension. Once the input energy is released, the dispersed droplets start to coalesce which drives the mixture back to equilibrium. The coalescence process can be diminished or stopped if amphiphilic materials (surfactants) exist on the interfacial surface between the phases[28]. When the surfactant coats the interface of the dispersed and continuous phase, the colloidal interaction forces between droplets change drastically when droplets get close to each other [29]. If the surfactant is non-ionic, flexible molecular chains protrude into the continuous phase and steric repulsion may appear. If the surfactant is ionic, repulsion also occurs through electrostatic forces 
[29]. This is how surfactants make the emulsion stable against coalescence.

Surfactants are either ionic or nonionic. Ionic surfactants can be cationic, anionic, or amphoteric. Surfactants contain a hydrophilic head and a hydrophobic tail. The hydrophilic heads of cationic, anionic, and amphoteric surfactants respectively have positively charged ions, negatively charged ions, and both types of ions at the same time. Non-ionic surfactants are categorized based on their Hydrophilic lipophilic balance (HLB) number which is the ratio of the molecular mass of the hydrophilic portion $\left(M_{h}\right)$ of the surfactant to the total molecular mass $(M)$. The HLB value for non-ionic surfactants is defined on a scale of 0 to 20 (HLB = $\left.20 \times M_{h} / M\right)$. Non-ionic surfactants with HLB $>10$ are used for direct emulsions and those with $\mathrm{HLB}<10$ are used for inverse emulsions. When the surfactant is nonionic, the addition of salts (ionic materials) to an aqueous solution increases the electrostatic repulsion and gives rise to emulsion stability.

\subsection{Rheology}

In spite of the fact that the emulsions are composed of fluids, they can show solid-like behavior and elasticity in case of high concentration of dispersed phase. This behavior exists because an external osmotic pressure $\Pi(\phi)$ squeezes the repulsive droplets together. The structure of droplets along with the state of their deformation and their interactions define the osmotic pressure at a given droplets volume fraction [30]. When the volume fraction of dispersed phase reaches the random close packing (rcp) which is $\phi^{r c p}=64 \%$ (mono-dispersed droplets), the osmotic pressure advances toward Laplace pressure $(2 \sigma / r)$, then droplets can no longer pack together without deformation. $(\sigma$ is interfacial tension and $r$ is the radius of undeformed droplet) Therefore, the increase of osmotic pressure causes more droplet deformation which increases the droplet surface area. This mechanism increases the elastic modulus of the material [30]. In this condition, when the emulsion is exposed to external shear stress, the time scale of droplet rearrangement is large enough that cannot relax the imposed stress quickly; therefore, the emulsions exhibit yield stress behavior $[31,32]$. However, when the osmotic pressure decreases below the Laplace pressure, the droplets will be unpacked and the emulsion does not have solid-like behavior anymore and becomes a viscous fluid.

There exist several models to explain the rheology of yield stress fluids such as the Herschel-Bulkley model which is a 1-D approximation. It expands the simple power-law model to include yield stress as the following.

$$
\begin{aligned}
\dot{\gamma}(u)=0 & \Longleftrightarrow \tau(u) \leq \tau_{y}(1) \\
\tau_{i j}(u)=\left(\frac{\tau_{y}}{\dot{\gamma}(u)}+k \dot{\gamma}(u)^{n-1}\right) \dot{\gamma}_{i j}(u) & \Longleftrightarrow \tau(u)>\tau_{y}(2)
\end{aligned}
$$

Where $\tau_{y}$ is the yield stress of the material, $k$ is the consistency index, and $n$ is the power law index. In all mentioned models, $\dot{\gamma}(u)$ and $\tau(u)$ denote the second invariant of strain rate and stress tensors respectively and therefore

$$
\begin{aligned}
& \dot{\gamma}_{i j}=\frac{\partial u_{i}}{\partial x_{j}}+\frac{\partial u_{j}}{\partial x_{i}} \\
& \dot{\gamma}(u)=\left[\frac{1}{2} \sum_{i, j=1}^{3}\left[\dot{\gamma}_{i j}(u)\right]^{2}\right]^{1 / 2} \\
& \tau(u)=\left[\frac{1}{2} \sum_{i, j=1}^{3}\left[\tau_{i j}(u)\right]^{2}\right]^{1 / 2}
\end{aligned}
$$

Rheological behavior of the yield stress emulsion is described here. Rheology tests are performed to characterize the nonlinear behavior of the material [33-35]. Test procedures are described, and the response of the fluid is explained in the following. A stress-controlled DHR-3 rheometer (TA Instruments) is used for rheology tests. A parallel plate (serrated) geometry with a gap of $2 \mathrm{~mm}$ is used for the tests. The temperature for all tests was set to be at $30^{\circ} \mathrm{C}$.

A peak hold test measures the yield stress of the material. For this test, the sample is pre-sheared by 20 $[1 / \mathrm{s}]$ for $30 \mathrm{~s}$ and then left to rest for $30 \mathrm{~s}$ to relax. The applied shear rate is $0.011 / \mathrm{s}$ for 7200 seconds. Figure 1 Shows the result of the peak hold test. The applied shear rate $(\dot{\gamma})$ is small enough to be able to measure the yield stress $\left(\tau_{y}\right)$ as the shear stress response of the rheometry test (see equation 2). Based on the peak hold test, two different regimes in material behavior are observed. The first regime is an approximately linear increment of the shear stress at the beginning of the test when the material has not yielded. This is due to the elastic behavior of the material which acts like a solid. The second regime is a stress plateau and occurs when the applied shear stress reaches the yield stress of the material (or when the applied strain reaches the yield strain). At this stage, the material has a fluid-like behavior and is characterized by its flow resistance.

A shear ramp test shows the magnitude of the yield stress, consistency index and power-law index of the material. A pre-shear of $20[1 / \mathrm{s}]$ is applied for $30 \mathrm{sec}-$ onds and then allowed to rest for 30 seconds to reach 
equilibrium. The decreasing shear ramp starts with 30 to $0.01[1 / \mathrm{s}]$. Figure 2 Shows the results of the shear ramp test. The Herschel-Bulkley model (equation 2) is fitted to this curve. All parameters of equation 2 can be derived from this test, and the magnitude of the yield stress $\left(\tau_{y}\right)$ shows a good agreement with the result in the peak hold test. For the case of simple yield stress fluids, the reproducibility of this test shows steady behavior of the material. If the material does not show the steady behavior, then it means the material is thixotropic.

Yield stress fluids usually have viscoelastic behavior in their solid states. In other words, when the strain is applied to the material, the elastic behavior is not instantaneous which shows the material exhibits viscous behavior. An amplitude sweep test helps to measure the elastic and viscous modulus of the material. When the yield stress fluid is in its linear regime, the elastic response of the material is in phase with the applied strain; However, the viscous response of the material is in phase with the applied strain rate. Consequently, applying oscillatory strain and measuring the response

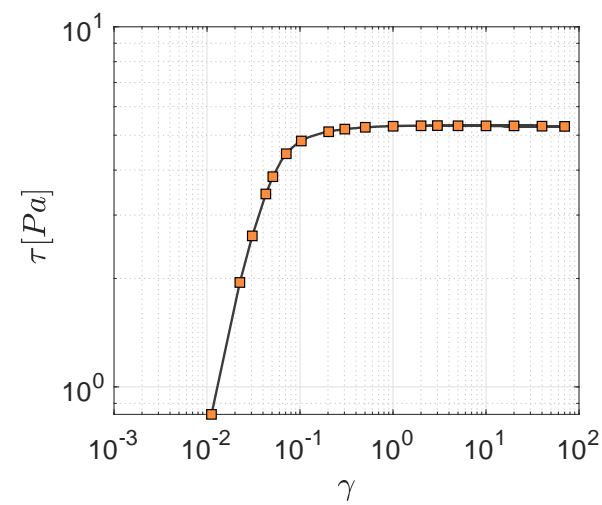

Fig. 1 Rheology result for peak-hold test to measure the yield stress of the material

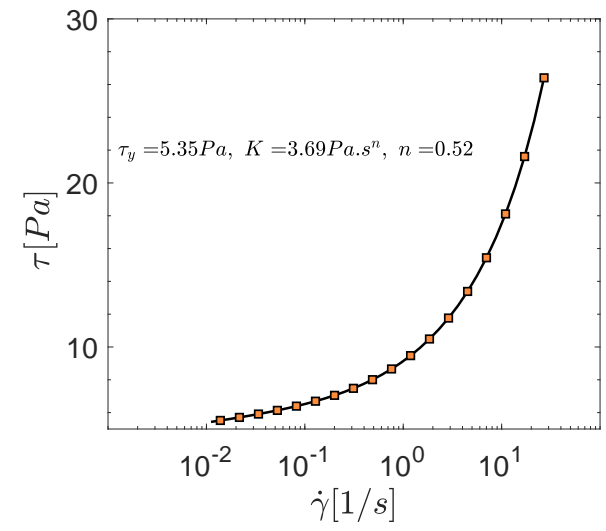

Fig. 2 Rheology result for shear-ramp test to measure the yield stress, consistency index, and the power law index of the material

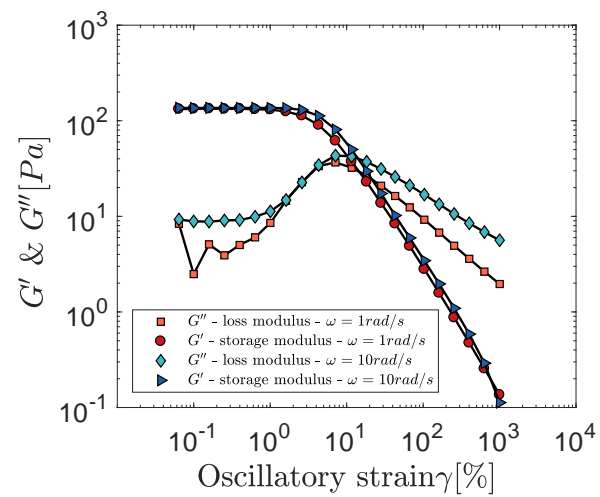

Fig. 3 Rheology result for amplitude sweep test to obtain the elastic and viscous modulus of the material with respect to strain amplitude

of the material helps to quantify the elastic (storage) and viscous (loss) modulus of the material. A pre-shear of $20[1 / \mathrm{s}]$ is applied for $30 \mathrm{~s}$ and allowed to rest for 30 seconds to reach equilibrium. Angular frequencies of $1 \mathrm{rad} / \mathrm{s}$ and $10 \mathrm{rad} / \mathrm{s}$ are chosen for the test. The range of strain is from 0.01 to $1000 \%$ for a logarithmic sweep with data acquisition of 5 points per decade. Figure 3 shows the magnitude of the viscous modulus $\left(G^{\prime \prime}\right)$ and elastic modulus $\left(G^{\prime}\right)$. The elastic modulus remains constant and is dominant below a critical strain which is the elastic regime of the material before reaching the yield stress. Once the material has yielded, the loss modulus is dominant. In other words, the applied energy to the material is mostly dissipated rather than being stored in the material.

In Figure 3 the amplitude sweep test was performed at two different frequencies and the storage modulus of the material shows the same order of magnitude in the linear regime for both cases. Now a frequency sweep test is performed to understand how the elastic and viscous modulus of the material responds to the frequency of the oscillatory strain. Based on the response of the material in Figure 3, four different oscillatory strains are chosen to perform the frequency sweep test $(\gamma=0.1,1$, 10 , and $100 \%$ ). This test shows how the elastic modulus of the material remains constant in its elastic regime. A pre-shear of $20[1 / \mathrm{s}]$ is applied for 30 seconds and allowed to rest for 30 seconds to reach equilibrium. Strains of $0.1,1,10$, and $100 \%$ at Angular frequencies 0.1 to $110 \mathrm{rad} / \mathrm{s}$ for a logarithmic sweep with data acquisition of 5 points per decade are chosen. Figure 4 shows at small amplitudes (e.g. $\bar{\gamma}=0.001$ ), the elastic response of the material is not a function of angular frequency and remains constant.

Mason et al. showed $\tau_{y} /(\sigma / r)$ only depends on the volume fraction of yield stress emulsions [36]. In other words, at a fixed volume fraction of droplets, the magni- 


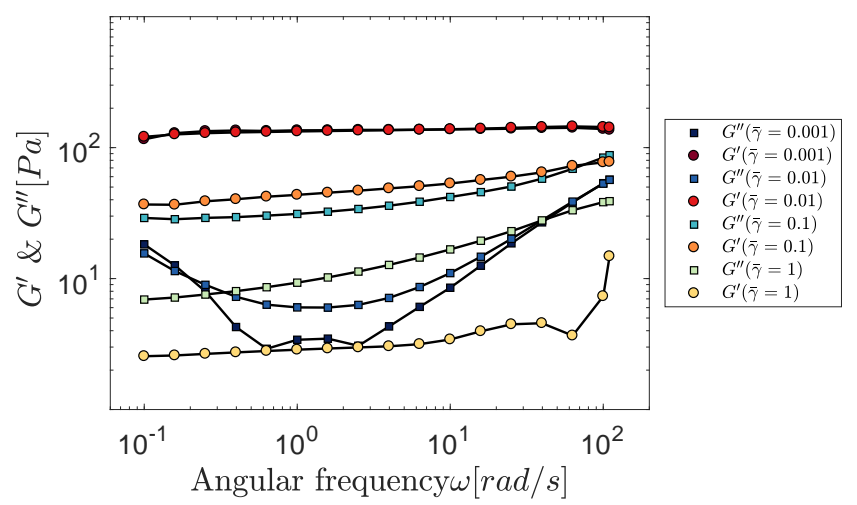

Fig. 4 Rheology result for frequency sweep test to obtain the elastic and viscous modulus of the material with respect to angular frequency of each strain amplitude

tude of $\tau_{y} /(\sigma / r)$ is constant. The droplet size $(r)$ of the dispersed phase is independent of the interfacial tension $(\sigma)$. Therefore, the interfacial tension is inversely related to the magnitude of the yield stress of the emulsion.

The consistency index depends on the volume fraction of the dispersed phase, viscosity of the phases and the interfacial tension (the effect of surfactants) [36]; However, changes in the volume fraction will change the yield stress and density of an emulsion as mentioned above. In addition, changing the materials in order to vary the viscosity of the phases will change the refractive index, yield stress, and density of an emulsion. Also, changing the surfactant affects the yield stress of the emulsion.

Olsson and Teitel comprehensively discussed the power law index appearing in the Herschel-Bulkley model [37]. A power law fit to $\tau-\tau_{y}$ as a function of the strain rate determines the magnitude of $n$. Numerical studies show two different power law patterns with two different magnitudes of $n$ [37-40]. They reported a power law index at low shear rates and high shear rates. Experimental studies show the second exponent (high shear rate) has more influence on the fit of the flow curves. At low shear rates there is a possibility of the existence of other effects like friction between droplets [41-43] or dissipated energy of interstitial liquid [44] which could change the magnitude of the power law index.

\subsection{Refractive index, Density, Rheology}

In order to design a transparent yield stress emulsion as a model fluid to perform experiments at different conditions, there is a need to show what range of refractive index, density, and rheology is possible to reach. The materials alternatives (o\&w) (e.g. choice of oil and water phase), volume fraction of the dispersed phase $(\phi)$, and the choice of surfactant (s) are important in the process of designing a transparent yield stress emulsion. The refractive index of the emulsion is a function of material alternatives (o\&w). The density of the emulsion is a function of material alternatives (o\&w) and the volume fraction of the dispersed phase $(\phi)$. The yield stress of the fluid $\left(\tau_{y}\right)$ is a function of material alternatives (o\&w), the volume fraction of the dispersed phase $(\phi)$, and the choice of surfactant (s). In other words equation 6 shows the properties of an emulsion as a function of the mentioned parameters.

$$
R I=f(o \& w) \quad \rho=f(o \& w, \phi) \quad \tau_{y}=f(o \& w, \phi, s)
$$

It means that the first step is to adjust the refractive index for both phases of an emulsion to the desired value. Then it is possible to change the volume fraction of the dispersed phase to adjust the density. At this stage, the yield stress magnitude of the fluid can be varied by the choice of surfactant. Note that surfactants with different HLB numbers will affect the consistency index as well which is explained in the following. In addition, in order to change the density without changing the refractive index, the materials for each phase and the volume fraction should change, however this might change the rheology of the fluid.

To design a stable transparent emulsion with given properties, it is necessary to go through several steps to achieve the target material [45]. Figure 5 shows the step by step design of the yield stress fluid with examples for direct and inverse emulsions. Our goal is to design a transparent yield stress emulsion with the refractive index of PMMA materials $(\mathrm{RI}=1.49)$. Table 1 lists the possible materials to be used to make transparent emulsions. According to Figure 5, the first step is to find proper materials (water phase, oil phase and surfactant) to make a stable emulsion, and it could be direct or inverse. We recommend silicon oil families for direct emulsions and mineral oils for inverse emulsions. The next step is to check if the refractive index of the water phase and the oil phase are matched together and close to the target value (e.g. $\mathrm{RI}=1.49$ ). If the $\mathrm{RI}$ does not match with the target value, then we check if there is a possibility of adding other materials. For example salts or alcohols can be added to change the RI of the water phase; or the derivatives of the oil phase can be added to adjust the RI of the oil phase. After the adjustment of the refractive index, the density can be tuned.

As mentioned earlier in this section, one method to adjust the density is to change the volume fraction of the dispersed phase. Another method is to choose different materials for the oil and the water phase which may also impact the refractive index. The other option 
to change the density is adding material to the chosen recipe (as mentioned before, salts or alcohols for water phase and oil phase derivatives for the oil phase).

The last step is to adjust the rheology of the fluid. One proposed method is to change the rheology via the surfactant. In the case of using non-ionic surfactants for direct emulsions, the higher the HLB number is, the higher yield stress and the consistency index is acquired. On the other hand, for the case of using non-ionic surfactants for inverse emulsions, the lower the HLB number is, the higher the yield stress and the consistency index will be. Other options to change the rheology of the fluid are to change or add material for each phase or change the volume fraction of the dispersed phase, keeping in mind that these changes might vary the refractive index and density of the final emulsion. In section 2.4 we introduce a transparent direct emulsion with $R I=1.49$ and $\rho=1.19 \mathrm{gr} / \mathrm{cm}^{3}$ with high yield stress $\left(\tau_{y} \sim 100 \mathrm{~Pa}\right)$ for this emulsion which is not compatible with PMMA material in a long term experiment (e.g. more than one day). Then in section 2.5 we introduce a transparent inverse emulsion that is RI and density matched with PMMA material and the yield stress is in order of $\left(\tau_{y} \sim 10 P a\right)$.

\subsection{Design a direct emulsion with $\mathrm{RI}=1.49$}

The first step is to start with an available, stable direct emulsion that is used in literature to study the flow in a non-Newtonian suspension. A dispersion of silicon oil in water with the aid of an ionic surfactant such as tetradecyltrimethylammonium bromide (TTAB) (Sigma-Aldrich 8.40006) makes a stable emulsion. Addition of glycerol (Sigma-Aldrich G5516) to the water phase (50\% vol.) makes it transparent with a refractive index of 1.40 for both phases [46]; However, glycerol will change the density of the emulsion.

Refractive index - It is essential to find correct immiscible phases with the same refractive index as PMMA materials. Some proposed oils with RI close to PMMA are oils (laser liquid (5610) $\rho=1: 034 \mathrm{gr} / \mathrm{ml} ; \mu=80 \mathrm{cS}$ $\mathrm{t} ; \mathrm{RI}=1: 4900$ ) and (immersion oil (5032) $\rho=0: 888$ $\mathrm{gr} / \mathrm{ml} ; \mu=10000 \mathrm{cS} \mathrm{t} ; \mathrm{RI}=1: 4917$ ) from Cargile Labs company and silicone 550 from Dow corning company $(\rho$ $=1: 064 \mathrm{gr} / \mathrm{ml} ; \mu=100 \mathrm{cS} \mathrm{t} ; \mathrm{RI}=1: 4985)$. The composition of these oils is Siloxane and is suitable for direct emulsions. All types of silicone oils (e.g. Gelest company) can be added to adjust the refractive index.

As mentioned before, water soluble/miscible materials such as salts, alcohols, and sugars (Table 1) can increase the refractive index of the water phase. A water solution of sugar (80\%) (Sigma-Aldrich S7903) has $\mathrm{RI}=1.49$, but it is not stable due to crystallization.
The refractive index of glycerol $(\mathrm{RI}=1.47)$ is lower than PMMA materials; other alcohols such as threitol (SigmaAldrich 377619), xylitol (Sigma-Aldrich X3375), and inositol (Sigma-Aldrich PHR1351) have higher refractive indices. However, their water solubility is low, and their solution cannot reach to $\mathrm{RI}=1.49$. Addition of salt such as $\mathrm{ZnCl} 2$ (Sigma-Aldrich 746355) in the water phase can increase the refractive index to 1.49. A comprehensive research on salts shows $\mathrm{ZnCl} 2$ is increasing the water phase's refractive index with the lowest impact on density.

Other liquids such as ethyl silicate (Sigma-Aldrich 86578), methyl silicate (Sigma-Aldrich 218472), ethyl cinnamate (Sigma-Aldrich 112372), and quinoline (SigmaAldrich 241571) have a high refractive index but these liquids are not easy to handle. In addition they react with the water and oil phases. Therefore it is not possible to use them for making stable emulsions.

Table 2 illustrates stable transparent direct emulsions with the refractive index of PMMA materials $(\mathrm{RI}=1.49)$.

Density - With the given materials listed in table 2, at the volume fraction of the dispersed phase $(\phi \sim 87 \%)$ the emulsion has the density of PMMA material. This high volume fraction of the dispersed phase causes high yield stress of the material. The other option is to add materials with different densities (e.g. adding glycerol to the water phase). Note this option might change the refractive index of the material as well.

Rheology - As mentioned before, changing the surfactant $(10<H L B<20)$ can vary the rheology of the material. The recipe of a direct emulsion that is RI and density matched with PMMA material is the following: oil phase: [Cargille oil-code $5610(67.98 \%$ wt. $)$ ], surfactant: [Tween $80(1.28 \%$ wt.)], water phase: [water(12.13\%wt.), $\mathrm{ZnCl} 2$ (24.31\%wt.)]. The rheology of the emulsion is $\tau=$ $104 P a, k=39.63 P a . s^{n}, n=0.47$. The Tween80 was purchased from Sigma-Aldrich (PX1289F).

Table 3 shows some of the examples of direct emulsion with different surfactants and different volume fractions.

\subsection{Design an inverse emulsion with $\mathrm{RI}=1.49$}

Dispersion of dodecane oil (Sigma-Aldrich 297879) in a water solution of $\mathrm{CaCl} 2$ (72\%) (Sigma-Aldrich 746495) with the aid of a non-ionic surfactant (span80) (SigmaAldrich 85548) produces a stable inverse emulsion [13]. This emulsion is designed to be density matched with polystyrene particles. However, it is not a transparent emulsion, but by increasing the concentration of $\mathrm{CaCl} 2$ in the water phase, it is possible to adjust the RI of both phases $(\mathrm{RI}=1.42)$. Increasing the salt concentration will change the density of the emulsion. 


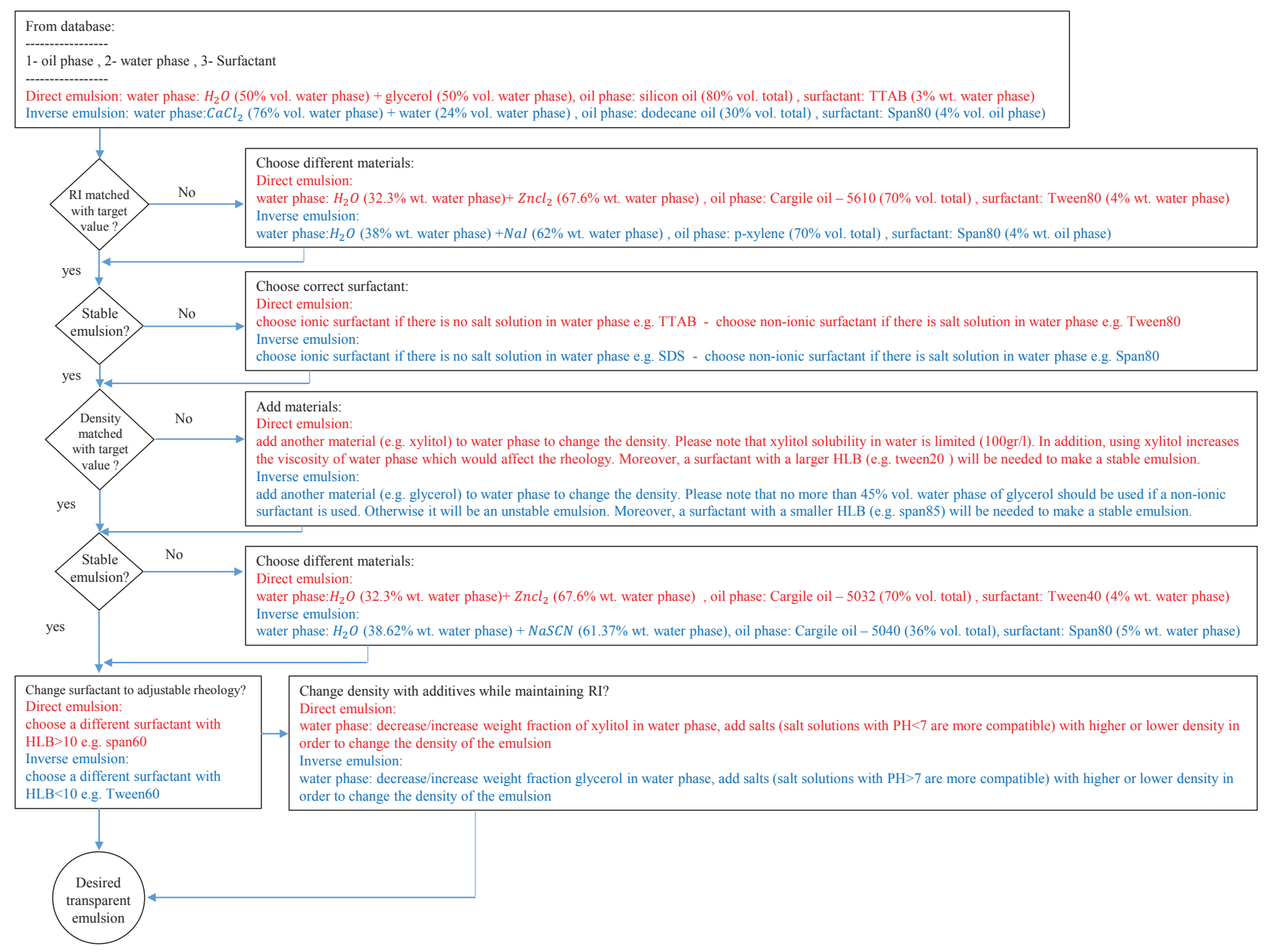

Fig. 5 Flow chart of making transparent yield stress emulsion with density and refractive index of PMMA materials

Refractive index - Hydrocarbon oils such as p-xylene (aromatic hydrocarbons) and Cargille immersion oil 5040 which is a combination of Aliphatic and Alicyclic Hydrocarbons and Hydrogenated Terphenyl $(\rho=0: 879$ $\mathrm{gr} / \mathrm{ml} ; \mu=27 \mathrm{cS}$ t; RI $=1: 4900$ ) are suitable for inverse emulsions. Note that P-xylene (Sigma-Aldrich 95680) is an aromatic hydrocarbon. It is not easy to handle in laboratory conditions and causes crazing on PMMA materials. For the water phase, addition of a salt such as NaI (Sigma-Aldrich 383112) or NaSCN (Sigma-Aldrich 467871) can increase the refractive index to 1.49. Table 2 illustrates stable, transparent direct emulsions with the refractive index of PMMA materials $(\mathrm{RI}=1.49)$.

Density - With the given materials listed in table 2, at the volume fraction of the dispersed phase $(\phi \sim 64 \%)$ the emulsion has the density of PMMA material. As another method to change the density, it is possible to add $\mathrm{NaI}$ or glycerol to the water phase to either drop or raise the density; However, note that the RI should be readjusted.
Rheology - As mentioned before, changing the surfactant $(0<H L B<10)$ can vary the rheology of the material.

\subsection{Instruction of making transparent yield stress} emulsions

The recipe of an RI and density matched (inverse) emulsion with PMMA that we chose is the following: Oil phase: [Cargille oil-code5040(25.46\%wt.)], surfactant: [Span80(1.34\%wt.)], water phase: [water(62.92\%wt.), NaSCN $(44.92 \%$ The Sodium thiocyanate Span80 (Sigma Aldrich 85548) is mixed with water at $T=60^{\circ} \mathrm{C}$ for two hours. The Span80 is mixed with Cargille oil-code 5040. The refractive index of both phases should be matched up to 4th digit $(\mathrm{RI}=1.4917)$. The materials are mixed together in a container surrounded by an ice water bath. A Silverson emulsifier L5M-A is used to mix the material. The rotational velocity of the emulsifier should increase gradually to make sure the materials are completely 
Table 1 Database of possible materials for making transparent yield stress emulsions with the density and refractive index of PMMA materials. Note that Salt solution with $P H<7$ are more compatible with direct emulsions. Also, Salt solution with $P H>7$ are more compatible with inverse emulsions. All silicone oil families can be use to adjust the refractive index the same family and are more recommended for the direct emulsions. All hydrocarbon oil families can be used to adjust the refractive index of the same family and are more recommended for the inverse emulsions. Non-ionic surfactant with $H L B>10$ are suitable for direct emulsions and Non-ionic surfactant with $H L B<10$ are suitable for inverse emulsions

\begin{tabular}{|c|c|c|c|c|c|c|c|c|}
\hline \multicolumn{4}{|c|}{ water solvents } & \multicolumn{2}{|c|}{$\begin{array}{l}\text { oil phases with refractive } \\
\text { index close to PMMA }\end{array}$} & \multicolumn{3}{|c|}{ surfactants } \\
\hline \multicolumn{3}{|c|}{$\begin{array}{l}\text { possible water } \\
\text { soluble salts }\end{array}$} & \multirow[t]{2}{*}{ alcohols } & \multirow[t]{2}{*}{$\begin{array}{l}\text { silicone } \\
\text { oil family }\end{array}$} & \multirow[t]{2}{*}{$\begin{array}{l}\text { mineral } \\
\text { oil family }\end{array}$} & \multirow[t]{2}{*}{$\begin{array}{l}\text { non-ionic } \\
\text { surfactants }\end{array}$} & \multicolumn{2}{|c|}{ ionic surfactants } \\
\hline cations & anions & examples & & & & & anionic & cationic \\
\hline$L i^{+}$ & $O H^{-}$ & $\mathrm{ZnCl} \mathrm{l}_{2}$ & $\begin{array}{l}\text { ethylene } \\
\text { glycol }\end{array}$ & Cargille-5610 & Cargille-5040 & tween family & SDS & TTAB \\
\hline$K^{+}$ & $F^{-}$ & $\mathrm{CaCl}_{2}$ & propylene & Cargille-5032 & p-xylene & span family & & CTAB \\
\hline $\mathrm{Na}^{+}$ & $C l^{-}$ & NaI & glycol & silicone550 & m-xylene & brij family & & \\
\hline$C s^{+}$ & $B r^{-}$ & $N a S C N$ & glycerol & & toluene & & & \\
\hline $\mathrm{NH}_{4}^{+}$ & $I^{-}$ & & threitol & & & & & \\
\hline$B a^{2+}$ & $S^{2-}$ & & xylitol & & & & & \\
\hline$C a^{2+}$ & $\mathrm{SO}_{3}^{2-}$ & & sorbitol & & & & & \\
\hline$M g^{2+}$ & $\mathrm{PO}_{4}^{2-}$ & & volemitol & & & & & \\
\hline$B a^{2+}$ & $S^{2-}$ & & $\begin{array}{l}\text { allyl } \\
\text { alcohol }\end{array}$ & & & & & \\
\hline$A l^{3+}$ & $\mathrm{CO}_{3}^{2-}$ & & geraniol & & & & & \\
\hline$C r^{3+}$ & $\mathrm{SiO}_{3}^{2-}$ & & propargyl & & & & & \\
\hline$F e^{2+}$ & $\mathrm{NO}^{-}$ & & $\begin{array}{l}\text { alcohol } \\
\text { inositol }\end{array}$ & & & & & \\
\hline$F e^{3+}$ & $\mathrm{CH}_{3} \mathrm{COO}^{-}$ & & menthol & & & & & \\
\hline$N i^{2+}$ & $S C N^{-}$ & & & & & & & \\
\hline $\mathrm{Co}^{2+}$ & & & & & & & & \\
\hline$M n^{2+}$ & & & & & & & & \\
\hline$Z n^{2+}$ & & & & & & & & \\
\hline $\mathrm{Ag}^{+}$ & & & & & & & & \\
\hline$H g^{2+}$ & & & & & & & & \\
\hline$R b^{2+}$ & & & & & & & & \\
\hline$S n^{2+}$ & & & & & & & & \\
\hline$C u^{2+}$ & & & & & & & & \\
\hline
\end{tabular}

Table 2 Ingredient combinations for direct and inverse emulsions that could be RI and Density matched with PMMA materials

\begin{tabular}{lll}
\hline Direct emulsions & & \\
\hline water phase & oil phase & $\begin{array}{l}\text { non-ionic } \\
\text { surfactant }\end{array}$ \\
\hline $\mathrm{H}_{2} \mathrm{O}+\mathrm{ZnCl}_{2}$ & silicon-550 & $(\mathrm{HLB}>10)$ \\
$\mathrm{H}_{2} \mathrm{O}+\mathrm{ZnCl} l_{2}$ & Cargille-5610 & $(\mathrm{HLB}>10)$ \\
$\mathrm{H}_{2} \mathrm{O}+\mathrm{ZnCl} l_{2}$ & Cargille-5032 & $(\mathrm{HLB}>10)$ \\
\hline Inverse emulsions & & \\
\hline water phase & oil phase & non-ionic \\
& & surfactant \\
\hline $\mathrm{H}_{2} \mathrm{O}+\mathrm{ZnCl} l_{2}$ & P-xylene & $(\mathrm{HLB}<10)$ \\
$\mathrm{H}_{2} \mathrm{O}+\mathrm{NaSCN} \mathrm{C}$ Cargille-5040 & $(\mathrm{HLB}<10)$ \\
\hline
\end{tabular}

mixed together and remain stable. It starts from 1000 rpm and increases $1000 \mathrm{rpm}$ every 10 minutes to reach $7000 \mathrm{rpm}$. A centrifuge is used to remove the trapped bubbles from the emulsion. After the centrifuge, a gra- dient of dispersed phase droplets will form in the microstructure of the emulsion. The emulsion is gently remixed to be completely homogeneous without trapping bubbles.

It is worthy to mention that for the direct emulsions, the surfactant should be mixed with the water phase before emulsification (e.g. water, $\mathrm{ZnCl}_{2}$, and Tween 80 will be mixed together at room temperature and then Cargille oil-code5610 will be added for emulsification).

\section{Applying transparent yield stress fluid suspension to study shear-induced migration}

To show the proposed emulsion is practical in studying suspension flows via an optical technique, we perform a typical test to measure the dispersion of solids in a heterogeneous shear field. This section describes our experimental setup, technique, and typical results. 
Table 3 Examples of direct emulsions. The oil phase is Cargille oil-5610 and the water phase is $(H 2 O(33.3 \% w t)+$. ZnCl2(66.7\%wt.))

\begin{tabular}{llllllll}
\hline water phase vol. & oil phase vol. & surfactant[4\%] vol. of water phase & $\tau_{y}[\mathrm{~Pa}]$ & $k\left[P a . s^{n}\right]$ & $n$ & $\rho\left[\mathrm{gr} / \mathrm{cm}^{3}\right]$ & $\mathrm{RI}$ \\
\hline $35 \%$ & $65 \%$ & Tween20 & 11.7 & 20.5 & 0.48 & 1.2776 & 1.4917 \\
$35 \%$ & $65 \%$ & Tween80 & 6.2 & 12.9 & 0.50 & 1.2776 & 1.4917 \\
$35 \%$ & $65 \%$ & Tween85 & 2.6 & 6.9 & 0.54 & 1.2776 & 1.4917 \\
$32.5 \%$ & $67.5 \%$ & Tween80 & 13.3 & 18.1 & 0.49 & 1.2602 & 1.4917 \\
$30 \%$ & $70 \%$ & Tween80 & 26.2 & 22.8 & 0.47 & 1.2428 & 1.4917 \\
\hline
\end{tabular}

\subsection{Experimental setup}

We study the shear-induced migration in a wide gap Taylor Couette flow. Several studies have been carried out to understand the physics of shear-induced migration. There exist two main approaches to characterize the migration in the flow of Newtonian suspensions. The first approach is called the "diffusive flux model" which considers diffusive terms in the mass balance of the particle phase $[47,48]$. This approach incorporates particle collision and relative viscosity gradients of the suspension to explain the migration. The second approach is called the "Suspension Balance Model" (SBM) which considers the suspension as a continuum media and takes into account the relative motion between the solid and fluid phase [49]. In this approach, particle normal stress gradients are responsible for particle flux [50]. Recently, Sarabian et al [50], experimentally investigated the migration in the flow of Newtonian fluid suspensions in a wide gap Taylor-Couette geometry via PTV techniques, and they showed the SBM is in good agreement with their results. They reported maximum migration occurs at $\phi \approx 35 \%$.

In spite of the fact that the suspensions of particles in yield stress fluids have more applications in industry, they have been less studied. Unlike Newtonian fluid suspension flow, the distribution of particles suspended in yield stress fluids is shear rate dependent[13].

Figure 6 shows the experimental setup. The Radii of the inner cylinder and outer cylinder are $R_{i}=5 \mathrm{~mm}$ and $R_{o}=9.5 \mathrm{~mm}$, respectively. The surface of inner cylinder is serrated to avoid slippage. The height of both cylinders is $70 \mathrm{~mm}$. The transparent and portable outer cylinder is a tube of PMMA and is located in a square water bath jacket coupled with a recirculator (Isotemp 4100R20 Bath-115V) to adjust the temperature of the whole set-up $\left( \pm 0 \cdot 1^{\circ} C\right)$. The outer cylinder is removable and can be directly placed in a centrifuge in order to remove the bubbles trapped in the suspension. A precision rotating stage (M-061.PD PI piezo-nano positioning) rotates the inner cylinder. A semiconductor green laser diode of $200 \mathrm{~mW}$ power and wavelength of $532 \mathrm{~nm}$ is used as the light source. The laser passes

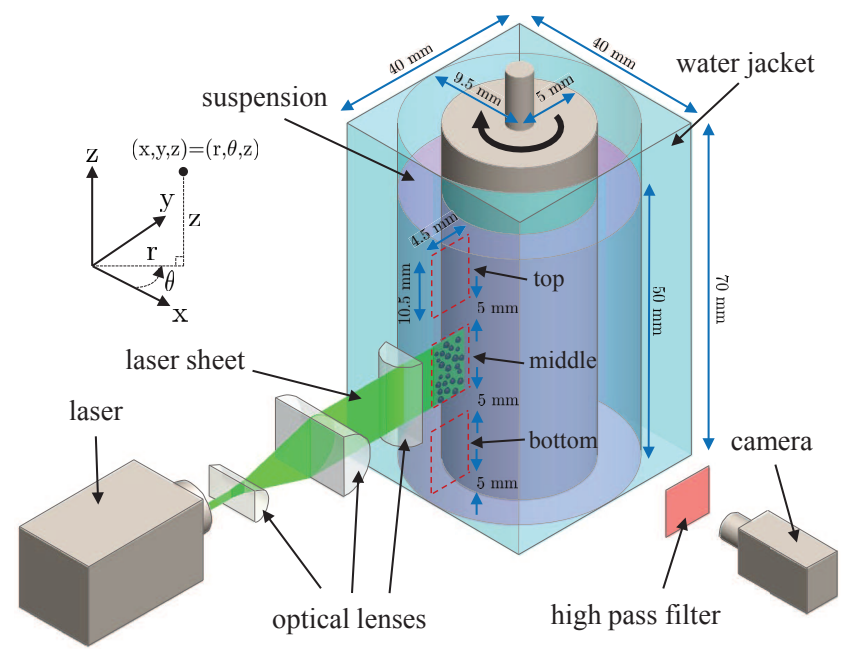

Fig. 6 Schematic view of experimental setup

through a horizontal plano-convex cylindrical lens with a focal length of $(f=10 \mathrm{~mm})$ to make a vertical laser sheet. The diverging laser sheet goes through a horizontal plano-convex cylindrical lens with a focal length of $(f=100 \mathrm{~mm})$ to collimate the laser sheet. A vertical plano-convex cylindrical lens with a focal length of $(f=50 \mathrm{~mm})$ is used to focus down the laser sheet thickness $(\approx 15 \mu \mathrm{m})[51,52]$. The suspending fluid contains rhodamine $6 \mathrm{G}$ with a concentration of $3 \mathrm{mg} / \mathrm{l}$, which illuminates under the laser diode projection. This concentration is obtained by trial and error to optimize the contrast between the particles and fluid all over the gap. The Particles that are used for experiments, are rigid PMMA spheres (Cospheric PMMAMS-1.2 355-425um) with density of $1.19 \mathrm{gr} / \mathrm{ml}$ and radius of $177-212 \mu \mathrm{m}$. The ratio between the Couette cell gap to particle size is thus $l_{\text {gap }} / a=\left(R_{o}-R_{i}\right) / a \approx 23$.

A digital camera (Basler Ace acA2000-165um USB3 Monochromatic) is implemented for Image acquisition. A macro lens (Sigma APO-Macro-180 mm-F3.5-DG) is connected to the camera via a $4 \mathrm{~cm}$ extension tube to enhance the magnification. A high-pass filter(550 nm) removes the noise and only allows light emitted by the fluorescent fluid to pass through and be projected on the lens. 
3.2 Migration of particles suspended in Newtonian fluids

First we calibrate our experimental setup and validate our results by performing one experiment on the shear induced migration of particles in a Newtonian suspending fluid which is a very well studied problem $[13,50$, 53].

The Newtonian suspending fluid is a viscous mixture. Water $(9.112 w t \%)$ and zinc chloride (14.561wt\%) are mixed together which is a Exothermic reaction. The solution temperature cools down to ambient temperature after 1 hour and then hydrochloric acid $(\mathrm{HCl})$ (0.085wt\%) (Sigma-Aldrich 320331) is added to enhance the transparency. Then, Triton X-100 (76.242wt\%) (SigmaAldrich X100) is added to the solution [54]. The viscosity of the prepared Newtonian fluid is 4.64 Pa.s at room temperature. The large viscosity of this Newtonian fluid helps to perform experiments at Reynolds number $\left(R e<10^{-3}\right)$. This Newtonian fluid is refractive index and the density matched with PMMA particles. The suspension of particles and Newtonian fluid is gently poured into the Couette cell. A centrifuge is used to remove bubbles from the suspension. After the centrifuge, the suspension is remixed gently with a small blade to make sure that the particle distribution is homogeneous. The temperature of the set-up is set to $12^{\circ} \mathrm{C} \pm 0.1^{\circ} \mathrm{C}$ to adjust the index matching of the particles and Newtonian fluid up to $10^{-4}$ ( $\left.\mathrm{RI}=1.4912\right)$.

For this case, the inner cylinder is rotating at 1.84 $\mathrm{rad} / \mathrm{s}$. The suspension was sheared over 10 minutes. The particles migrated toward the outer cylinder over time. The bulk solid volume fraction for all the experiments is set to be $35 \%$ since the maximum migration is reported to be at $\phi=35 \%$ [50]. Figure 7 (a) shows the raw image captured by the PTV technique. The laser beam intensity is Gaussian, and therefore the intensity of the laser sheet is not uniform. In addition, the intensity drops as the laser penetrates through the suspension due to a slight refractive index mismatch. The intensity profile of the raw image is normalized by a reference image. The reference image is obtained by applying a Gaussian blur filter (50 pixel in width and height) on the same image. This procedure helps to obtain a uniform image with a wide spatial range of contrast and intensity level [55]. Figure 7 (b) illustrates the successive threshold of the normalized image.

The suspension is initially homogeneous, so the gradient of $\phi$ in the gap is zero. Consequently, the velocity profile of the Newtonian suspension at the beginning of the experiment is the same as if there were no particles in the system. So the theoretical expression of shear rate resulting from the solution of the Navier-Stokes equa-

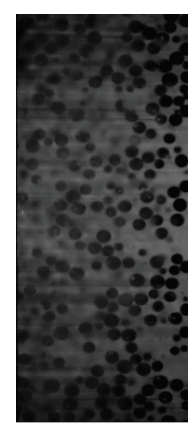

(a)

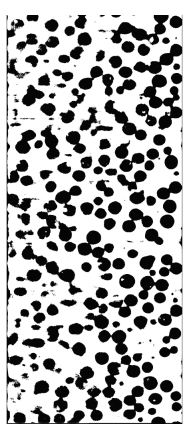

(b)

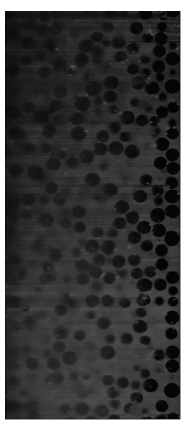

(c)

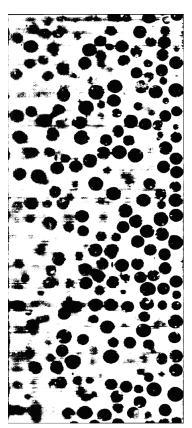

(d)
Fig. 7 (a) Raw image of Newtonian suspension, (b) Thresholding of Newtonian suspension, (c) Raw image of emulsion suspension, (d) Thresholding of emulsion suspension

tion in the Taylor-Couette geometry of pure Newtonian fluid, is used to calculate the shear rate (Equation 7).

$\dot{\gamma}=\frac{2 R_{o}^{2} \Omega}{R_{o}^{2}-R_{i}^{2}}$

Based on the shear rate, the time step (at each strain unit) for image acquisition is defined. For each experiment, we take 2630 images from the middle of the Couette cell. Then 100 images are taken from the top and the bottom sections. Figure 6 clearly shows the exact location of each target window $(4.5 \mathrm{~mm} \times 10.5 \mathrm{~mm})$ for each location. The average over 100 images at steadystate conditions for each location is reported. At the beginning of the experiment, we made sure the suspension was homogeneous all over the cell with minimum deformation of the suspension in order to uniformly distribute the solid particles.

Figure 8 shows the color-map for the distribution of the solid volume fraction in the Newtonian fluid at steady-state conditions for the top, middle, and bottom of the cell, and we observed no sedimentation or rise up. In order to be far from the boundary effects, the radial distribution of the solid volume fraction for the middle window is compared with the SBM. We note that the particles have migrated from the inner cylinder to the outer cylinder resulting in a large volume fraction at the outer wall.

In order to generate the color-map, the average of each batch of 100 images is divided into overlapping sub-images with the size of two-particle diameters $(120 \times 120$ pixels). The radial distributions are obtained by a vertical average over the threshold of 100 images at the steady-state condition. The fluctuations on the radial distribution of a solid volume fraction is the result of the layering of particles due to the confinement effect.

Figure 8.b shows that our measurements are in agreement with the SBM results, and therefore our experimental setup can be used to study the shear-induced migration of particles in yield stress fluids. 

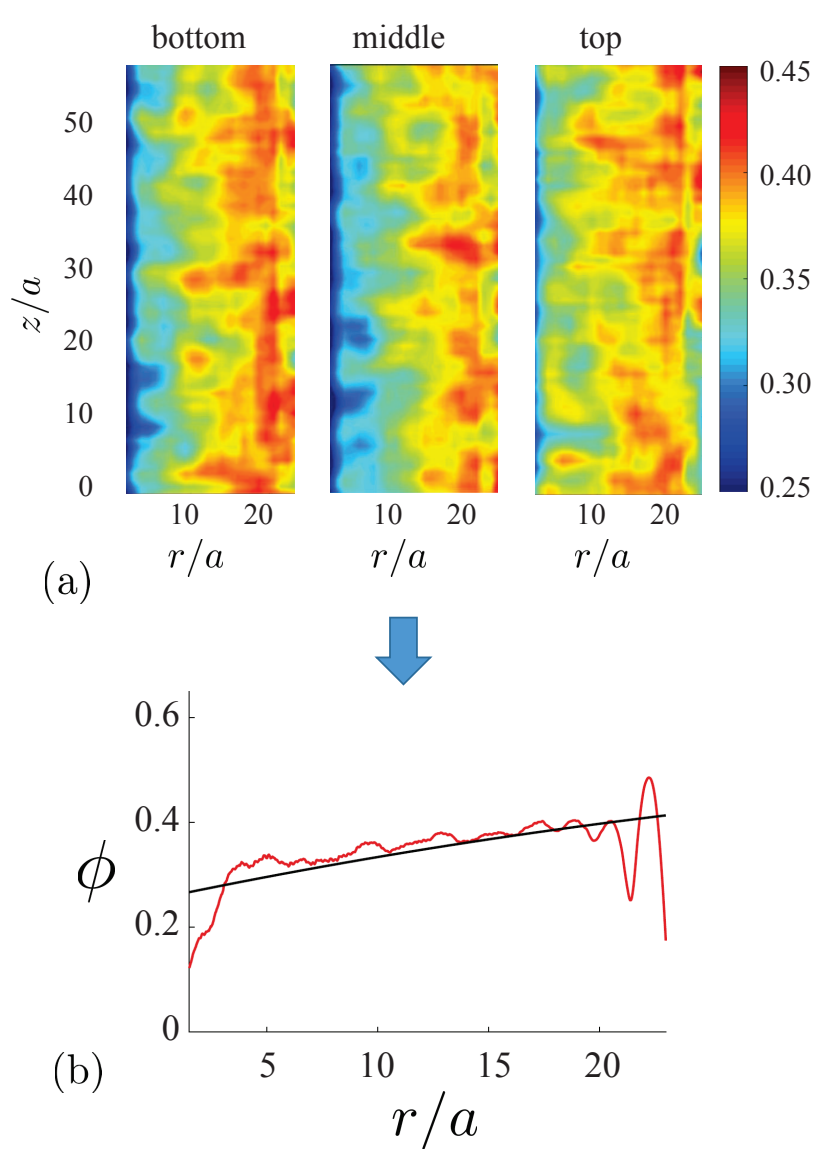

Fig. 8 (a) Color-maps for the distribution of the solid volume fraction suspended in Newtonian fluid at steady-state conditions for different positions of the cell - (b) Distribution of the solid volume fraction at the steady state condition at the middle of the Taylor-Couette cell for a Newtonian fluid suspension $(\Omega=1.48 \mathrm{rad} / \mathrm{s})$ (red line), suspension balance model (black line) [56]

\subsection{Migration of particles suspended in yield stress fluid}

The suspending fluid for the non-Newtonian experiment is a yield stress transparent emulsion, as it was mentioned in section 2.3, composed of a water phase (53.47wt\% NaSCN and 33.65wt\% DI water) and an oil phase (25.46wt\% Cargille oil code 5040 and $1.34 w t \%$ span 80 as surfactant). The materials and weight percentage are set to make a stable, transparent yield stress emulsion with the refractive index and density of PMMA materials. After mixing PMMA particles with the emulsion, the suspension is placed in a centrifuge (at 1350 g) to remove the bubbles. Then the suspension is mixed gently to homogenize the particle distribution and remove the gradient of the water phase droplets in the emulsion. The suspension is delicately poured into the couette geometry. The couette geometry is placed in the centrifuge (at $110 \mathrm{~g}$ ) to remove any possible large bubbles without displacing the PMMA particles. Before performing the experiment, we made sure the particles are homogeneously distributed in the Couette cell.

The temperature of the set-up is set to $30^{\circ} \mathrm{C} \pm 0.1^{\circ} \mathrm{C}$ to match the refractive index of the yield stress fluid and PMMA particles (within $10^{-4}$ ). In fact, rheology tests in section 2.2 were performed at $30^{\circ} \mathrm{C}$ because the yield stress fluid is index matched with PMMA particles at this temperature. Purposefully, the refractive index of the transparent yield stress emulsion is set to be the same as PMMA particles when close to room temperature. Considerable change in temperature might affect the transparency of the fluid. This is due to the fact that the refractive index of the oil and water phases of the emulsion are different functions of temperature $[18$, $57]$.

Here we show the results for the migration of particles suspended in yield stress fluids. Since the shear stress $(\tau)$ decreases radially in wide gap Taylor-Couette flow $\left(\tau \propto T / r^{2}\right)$. Where $T$ is the applied torque and $r$ is the radius. The yield stress fluid suspension follows the Herschel-Bulkley model [58]. Therefore, when the applied shear rate is more than the yield stress of the suspension $\left(\tau \geq \tau_{y}\right)$, the material flows. Otherwise it behaves as a solid. Figure 9 shows the schematic view for the flow of the yield stress fluid suspension in the Couette cell. For the yield stress fluid suspension, the rotating speed of the inner cylinder is considered at two different conditions to observe fully yielded and partially yielded conditions. For the fully yielded condition, the material was sheared at $1.48 \mathrm{rad} / \mathrm{s}$ for 10 minutes. For the partially yielded condition, the material was sheared at $0.006 \mathrm{rad} / \mathrm{s}$ for 42 hours.

Figure 7 (c) shows the raw image that captured the suspension particles in the yield stress fluid. Figure 7 (d) illustrates the successive threshold of the normalized image as it was discussed in section 3.2. Comparison of Figures 7 (a) and (c) demonstrates the quality of the experiments and how accurately the refractive indices of the solid and liquid phases are matched together.

Figure 10.a shows the color-map for the distribution of the solid volume fraction in the yield stress fluid at a steady state condition for the fully yielded suspension flow. Over time the particles have migrated toward the outer cylinder. Figure 10.b shows the color-map for the distribution of the solid volume fraction in the yield stress fluid at a the steady state condition for the partially yielded suspension flow. The migration occurs only until $r / a=10$, and then the flat volume fraction profile is consistent with the existence of plug in the gap. The color-map shows that the particles accumu- 


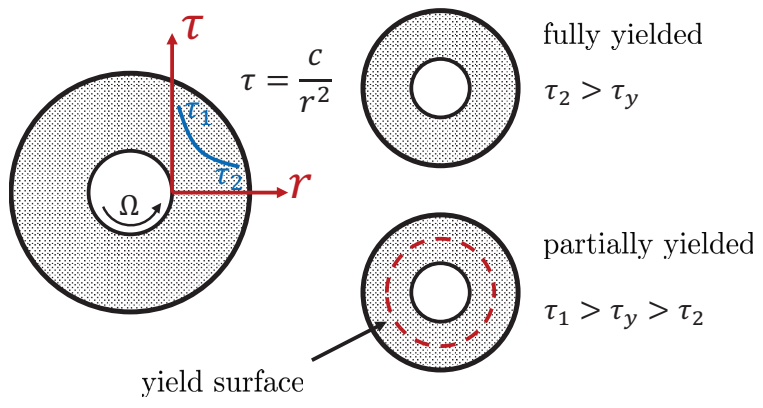

Fig. 9 Schematic view of shear stress profile in a large gap Couette cell containing viscoplastic material

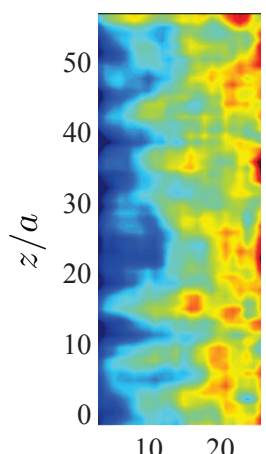

(a) $r / a$

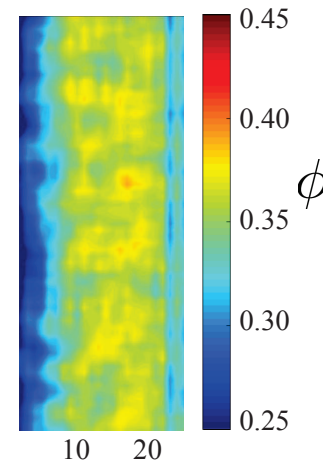

(b) $r / a$

Fig. 10 Color-maps for the distribution of solid volume fraction suspended in yield stress fluid at steady state condition for (a) the fully yielded suspension and (b) the partially yielded condition

late at the yield surface and they do not penetrate in the plug.

\section{Summary}

We introduced the design procedure of making transparent yield stress emulsions (direct/inverse) with the same refractive index and density of PMMA materials. It can be used to study the dynamic flow of complex suspensions in many natural and industrial problems using optical methods such as Particle Tracking Velocimetry or Laser Doppler Velocimetry, which are by far more affordable and accessible compared to other methods such as X-ray Radiography and Magnetic Resonance Imaging. We have provided typical examples to show the shear induced migration of particles in yield stress fluids. The objective of this paper is to introduce the design of this model suspension to the community. In the future, we will provide a detailed study on the modeling and experimental measurements of shear induced migration of particles in yield stress fluids.

Acknowledgements This work was supported by NSF (Grant no. CBET-1554044-CAREER) and ACS PRF (Grant no. 55661-
DNI9) via the research awards (S.H.) and the support of IdEx - University of Bordeaux. Fruitful discussions with Mohammad Sarabian are acknowledged. We acknowledge Amir Farnoud for providing circulating water bath facility.

\section{References}

1. Neil J Balmforth, Ian A Frigaard, and Guillaume Ovarlez. Yielding to stress: recent developments in viscoplastic fluid mechanics. Annual Review of Fluid Mechanics, 46:121-146, 2014.

2. Ahmadreza Rashedi. A Study of Surface Wetting in OilWater Flow in Inclined Pipeline. PhD thesis, Ohio University, 2016.

3. Robert L Powell. Experimental techniques for multiphase flows. Physics of fluids, 20(4):040605, 2008.

4. Sébastien Manneville, Lydiane Bécu, and Annie Colin. High-frequency ultrasonic speckle velocimetry in sheared complex fluids. The European Physical Journal-Applied Physics, 28(3):361-373, 2004.

5. Thomas Gallot, Christophe Perge, Vincent Grenard, Marc-Antoine Fardin, Nicolas Taberlet, and Sébastien Manneville. Ultrafast ultrasonic imaging coupled to rheometry: Principle and illustration. Review of Scientific Instruments, 84(4):045107, 2013.

6. Guillaume Ovarlez, François Bertrand, and Stéphane Rodts. Local determination of the constitutive law of a dense suspension of noncolloidal particles through magnetic resonance imaging. Journal of rheology, 50(3):259$292,2006$.

7. Guillaume Ovarlez, Fabien Mahaut, François Bertrand, and Xavier Chateau. Flows and heterogeneities with a vane tool: Magnetic resonance imaging measurements. Journal of rheology, 55(2):197-223, 2011.

8. Stéphane Rodts, Jalila Boujlel, Brooks Rabideau, Guillaume Ovarlez, Nicolas Roussel, Pascal Moucheront, Christophe Lanos, François Bertrand, and Philippe Coussot. Solid-liquid transition and rejuvenation similarities in complex flows of thixotropic materials studied by $\mathrm{nmr}$ and mri. Physical Review E, 81(2):021402, 2010.

9. JS Raynaud, P Moucheront, JC Baudez, F Bertrand, JP Guilbaud, and Ph Coussot. Direct determination by nuclear magnetic resonance of the thixotropic and yielding behavior of suspensions. Journal of Rheology, 46(3):709-732, 2002.

10. KG Hollingsworth, AJ Sederman, C Buckley, LF Gladden, and ML Johns. Fast emulsion droplet sizing using nmr self-diffusion measurements. Journal of colloid and interface science, 274(1):244-250, 2004.

11. RE Hampton, AA Mammoli, AL Graham, N Tetlow, and SA Altobelli. Migration of particles undergoing pressuredriven flow in a circular conduit. Journal of Rheology, 41(3):621-640, 1997.

12. Guillaume Ovarlez, François Bertrand, Philippe Coussot, and Xavier Chateau. Shear-induced sedimentation in yield stress fluids. Journal of Non-Newtonian Fluid Mechanics, 177:19-28, 2012.

13. Guillaume Ovarlez, Fabien Mahaut, Stéphanie Deboeuf, Nicolas Lenoir, Sarah Hormozi, and Xavier Chateau. Flows of suspensions of particles in yield stress fluids. Journal of rheology, 59(6):1449-1486, 2015.

14. Alana M Corbett, Ronald J Phillips, Robert J Kauten, and Kathryn L McCarthy. Magnetic resonance imaging of concentration and velocity profiles of pure fluids and solid suspensions in rotating geometries. Journal of Rheology, 39(5):907-924, 1995. 
15. Willi A Kalender. X-ray computed tomography. Physics in Medicine \& Biology, 51(13):R29, 2006.

16. Theodore $\mathrm{J}$ Heindel. A review of $\mathrm{x}$-ray flow visualization with applications to multiphase flows. Journal of Fluids Engineering, 133(7):074001, 2011.

17. Mohammad Gholami, Ahmadreza Rasehdi, Nicolas Lenoir, David Hautemayou, Guillaume Ovarlez, and $\mathrm{S}$ Hormozi. Time-resolved $2 \mathrm{~d}$ concentration maps in flowing suspensions using x-ray. J. Rheol., 2018.

18. Sébastien Wiederseiner, Nicolas Andreini, Gaël EpelyChauvin, and Christophe Ancey. Refractive-index and density matching in concentrated particle suspensions: a review. Experiments in fluids, 50(5):1183-1206, 2011.

19. MK Lyon and LG Leal. An experimental study of the motion of concentrated suspensions in two-dimensional channel flow. part 1. monodisperse systems. Journal of Fluid Mechanics, 363:25-56, 1998.

20. MK Lyon and LG Leal. An experimental study of the motion of concentrated suspensions in two-dimensional channel flow. part 2. bidisperse systems. Journal of Fluid Mechanics, 363:57-77, 1998.

21. Frédéric Blanc. Rhéologie et microstructure des suspensions concentrées non browniennes. $\mathrm{PhD}$ thesis, Université Nice Sophia Antipolis, 2011.

22. Mathieu Souzy, Henri Lhuissier, Emmanuel Villermaux, and Bloen Metzger. Stretching and mixing in sheared particulate suspensions. Journal of Fluid Mechanics, 812:611-635, 2017.

23. Margaret L Byron and Evan A Variano. Refractive-indexmatched hydrogel materials for measuring flow-structure interactions. Experiments in fluids, 54(2):1456, 2013.

24. S Sinha-Ray, K Fezzaa, and AL Yarin. The internal structure of suspensions in uniaxial elongation. Journal of Applied Physics, 113(4):044906, 2013.

25. Suman Sinha-Ray, Raman Srikar, Chris C Lee, Alfred Li, and Alexander L Yarin. Shear and elongational rheology of gypsum slurries. Applied Rheology, 21(6):1-8, 2011.

26. Manish K Tiwari, Alexander V Bazilevsky, Alexander L Yarin, and Constantine M Megaridis. Elongational and shear rheology of carbon nanotube suspensions. Rheologica acta, 48(6):597-609, 2009.

27. AL Yarin, E Zussman, A Theron, S Rahimi, Z Sobe, and D Hasan. Elongational behavior of gelled propellant simulants. Journal of Rheology, 48(1):101-116, 2004.

28. Rodrigo Emigdio Guerra. Elasticity of compressed emulsions. PhD thesis, 2014

29. Daniel Schuster. Encyclopedia of emulsion technology, volume 4. CRC Press, 1996.

30. TG Mason, Martin-D Lacasse, Gary S Grest, Dov Levine, $\mathrm{J}$ Bibette, and DA Weitz. Osmotic pressure and viscoelastic shear moduli of concentrated emulsions. Physical Review E, 56(3):3150, 1997.

31. M Dinkgreve, MAJ Michels, TG Mason, and D Bonn. Crossover between athermal jamming and the thermal glass transition of suspensions. Physical Review Letters, 121(22):228001, 2018.

32. Daniel Bonn, Morton M Denn, Ludovic Berthier, Thibaut Divoux, and Sébastien Manneville. Yield stress materials in soft condensed matter. Reviews of Modern Physics, 89(3):035005, 2017.

33. Philippe Coussot, H Tabuteau, Xavier Chateau, L Tocquer, and G Ovarlez. Aging and solid or liquid behavior in pastes. Journal of Rheology, 50(6):975-994, 2006.

34. Christopher W Macosko and Ronald G Larson. Rheology: principles, measurements, and applications. 1994.

35. QD Nguyen and DV Boger. Measuring the flow properties of yield stress fluids. Annual Review of Fluid Mechanics, 24(1):47-88, 1992.
36. TG Mason, J Bibette, and DA Weitz. Yielding and flow of monodisperse emulsions. Journal of Colloid and Interface Science, 179(2):439-448, 1996.

37. Peter Olsson and S Teitel. Critical scaling of shearing rheology at the jamming transition of soft-core frictionless disks. Physical Review E, 83(3):030302, 2011.

38. Peter Olsson and S Teitel. Herschel-bulkley shearing rheology near the athermal jamming transition. Physical review letters, 109(10):108001, 2012.

39. Edan Lerner, Gustavo Düring, and Matthieu Wyart. A unified framework for non-brownian suspension flows and soft amorphous solids. Proceedings of the National Academy of Sciences, 2012.

40. Takeshi Kawasaki, Daniele Coslovich, Atsushi Ikeda, and Ludovic Berthier. Diverging viscosity and soft granular rheology in non-brownian suspensions. Physical Review E, 91(1):012203, 2015.

41. Gijs Katgert, Matthias E Möbius, and Martin van Hecke. Rate dependence and role of disorder in linearly sheared two-dimensional foams. Physical review letters, 101(5):058301, 2008.

42. Gijs Katgert, Andrzej Latka, Matthias E Möbius, and Martin van Hecke. Flow in linearly sheared twodimensional foams: From bubble to bulk scale. Physical Review E, 79(6):066318, 2009.

43. Roger T Bonnecaze and Michel Cloitre. Micromechanics of soft particle glasses. In High Solid Dispersions, pages 117-161. Springer, 2010.

44. LW Schwartz and HM Princen. A theory of extensional viscosity for flowing foams and concentrated emulsions. Journal of colloid and interface science, 118(1):201-211, 1987.

45. Arif Z Nelson and Randy H Ewoldt. Design of yield-stress fluids: a rheology-to-structure inverse problem. Soft matter, 13(41):7578-7594, 2017.

46. Jalila Boujlel, Mathilde Maillard, A Lindner, Guillaume Ovarlez, Xavier Chateau, and Philippe Coussot. Boundary layer in pastes - displacement of a long object through a yield stress fluid. Journal of rheology, 56(5):1083-1108, 2012.

47. David Leighton and Andreas Acrivos. The shear-induced migration of particles in concentrated suspensions. Journal of Fluid Mechanics, 181:415-439, 1987.

48. Ronald J Phillips, Robert C Armstrong, Robert A Brown, Alan L Graham, and James R Abbott. A constitutive equation for concentrated suspensions that accounts for shear-induced particle migration. Physics of Fluids A: Fluid Dynamics, 4(1):30-40, 1992.

49. Prabhu R Nott and John F Brady. Pressure-driven flow of suspensions: simulation and theory. Journal of Fluid Mechanics, 275:157-199, 1994.

50. Mohammad Sarabian, Mohammadhossein Firouznia, Bloen Metzger, and Sarah Hormozi. Fully developed and transient concentration profiles of particulate suspensions sheared in a cylindrical couette cell. Journal of Fluid Mechanics, 862:659-671, 2019.

51. G Diemunsch and JP Prenel. A compact light sheet generator for flow visualizations. Optics \& Laser Technology, 19(3):141-144, 1987.

52. JP Prenel and M Jeudy. A new versatile laser sheet generator for flow visualization. Optics 83 Laser Technology, 30(8):533-538, 1998.

53. Mohammad Gholami, Ahmadreza Rashedi, Nicolas Lenoir, David Hautemayou, Guillaume Ovarlez, and Sarah Hormozi. Time-resolved 2d concentration maps in flowing suspensions using x-ray. Journal of Rheology, 62(4):955-974, 2018. 
54. Phong Ngoc Pham. Origin of shear-induced diffusion in particulate suspensions: crucial role of solid contacts between particles. PhD thesis, Aix-Marseille, 2016.

55. Frédéric Blanc, Elisabeth Lemaire, Alain Meunier, and François Peters. Microstructure in sheared nonbrownian concentrated suspensions. Journal of rheology, 57(1):273-292, 2013.

56. Jeffrey F Morris and Fabienne Boulay. Curvilinear flows of noncolloidal suspensions: The role of normal stresses. Journal of rheology, 43(5):1213-1237, 1999.

57. Joshua A Dijksman, Frank Rietz, Kinga A Lörincz, Martin van Hecke, and Wolfgang Losert. Invited article: Refractive index matched scanning of dense granular materials. Review of Scientific Instruments, 83(1):011301, 2012.

58. Simon Dagois-Bohy, Sarah Hormozi, Élisabeth Guazzelli, and Olivier Pouliquen. Rheology of dense suspensions of non-colloidal spheres in yield-stress fluids. Journal of Fluid Mechanics, 776, 2015. 